\title{
DESENVOLVIMENTO DE UMA FERRAMENTA DE PARAMETRIZAÇÃO DE UM PROJETO MECÂNICO/ESTRUTURAL E CUSTO DE UMA CHAMINÉ DE GASES
}

\author{
DEVELOPMENT OF A PARAMETERIZATION TOOL FOR A \\ MECHANICAL/STRUCTURAL PROJECT AND COST OF A GAS CHIMNEY
}

\author{
Kleber Augusto Rama Sato \\ Rodolfo Rodrigo de Souza Lauriano ${ }^{\mathrm{II}}$ \\ Maria Aparecida Bovério ${ }^{\mathrm{III}}$ \\ Antônio Carlos Muniz Ventura Júnior ${ }^{\mathrm{IV}}$
}

\begin{abstract}
RESUMO
Com a difusão da parametrização na indústria, ela se tornou uma ferramenta de grande valia, pois, por meio dela, se obtém a redução de custo em relação a engenheiros e técnicos. Esse artigo apresenta um projeto que desenvolve a parametrização e o custo de uma chaminé de gases, com a utilização do software Autodesk Inventor para elaboração da parametrização, desenho e modelagem, e o software Excel, para cálculos, realizados por fórmulas e funções construídas pelos autores, tendo, assim, a elaboração do custo estimado. Observou-se que os setores de engenharia e custo utilizam metodologias de elaboração de projeto e levantamento de custos distintos. Tais fatores internos se definem pelo feeling e o know-how de cada profissional. O tema foi escolhido considerando-se a necessidade de integração entre os setores de custo e engenharia de produtos, com o propósito de reduzir o tempo de trabalho e retrabalho. Os resultados apresentam todas as possibilidades de ambos os softwares. Concluiu-se que no método de trabalho tradicional leva em torno de 4 a 5 dias para o estudo do projeto, levantamento de materiais e de custos. E, após o desenvolvimento dessa ferramenta, esse tempo cai para em torno de 1 a 2 horas, o que é bastante significativo.
\end{abstract}

Palavras-chave: Parametrização. Custos. Chaminé de Gases.

\begin{abstract}
With the diffusion of parameterization in the industry, it has become a tool of great value, because, through it, it obtains cost reduction in relation to engineers and technicians. This article presents a project that develops the parameterization and cost of a gas chimney, with the use of Autodesk Inventor software for the elaboration of parameterization, design, and modeling, and excel software, for calculations, performed by formulas and functions built by

\footnotetext{
I Estudante do curso superior de Tecnologia em Mecânica: processos de soldagem da Faculdade de Tecnologia Deputado Waldyr Alceu Trigo (Fatec) de Sertãozinho - SP - Brasil. E-mail: kleber_sato@hotmail.com

II Estudante do curso superior de Tecnologia em Mecânica: processos de soldagem da Faculdade de Tecnologia Deputado Waldyr Alceu Trigo (Fatec) de Sertãozinho - SP - Brasil. E-mail: rodolfosueli@gmail.com

III Pós-Doutorado em Educação (UNESP-Rio Claro), Doutorado, Mestrado e Especialização em Educação (UNESP-Araraquara), Bacharel em Letras (Centro Universitário Moura Lacerda-Ribeirão Preto). Docente e pesquisadora da Faculdade de Tecnologia (Fatec) - Câmpus de Sertãozinho e Jaboticabal. Membro do Grupo de Estudos e Pesquisas em Políticas e Gestão da Educação Superior (GEPES) do Departamento de Educação da UNESP, Câmpus de Rio Claro, vinculado ao CNPq. E-mail: maria.boverio@fatec.sp.gov.br

IV Profa. Me. da Faculdade de Tecnologia Deputado Waldyr Alceu Trigo (Fatec) de Sertãozinho - SP - Brasil. E-mail: antonio.ventura@fatec.sp.gov.br
} 
the authors, thus having the elaboration of the estimated cost. It was observed that the engineering and cost sectors use methodologies for project elaboration and different cost survey. These internal factors are defined by the feeling and know-how of each professional. The theme was chosen considering the need for integration between the cost and product engineering sectors, with the purpose of reducing working time and rework. The results present all the possibilities of both softwares. It was concluded that in the traditional working method it takes around 4 to 5 days to study the project, survey of materials and costs. And after the development of this tool, this time drops to around 1 to 2 hours, which is quite significant.

Keywords: Parameterization. Costs. Gas chimney.

Data de submissão do artigo: 06/04/2021.

Data de aprovação do artigo: 13/05/2021.

DOI: $10.33635 /$ sitefa.v4i1.149

\section{INTRODUÇÃO}

Esse artigo apresenta os resultados de uma pesquisa de revisão bibliográfica, seguida de uma aplicada, cujo objetivo foi desenvolver um projeto de parametrização de uma chaminé de gases que pode ser utilizada em caldeiras, no mercado de açúcar e álcool, petroquímico, papel e celulose entre outros.

Nesse contexto, o objeto dessa pesquisa, assim como a sua delimitação, é o estudo e desenvolvimento do projeto, não sua implementação prática. Para isso, foi, inicialmente, calculado o custo e, em seguida, a partir do projeto básico foi feito o projeto final nos softwares Excel e Autodesk Inventor.

O tema foi escolhido considerando-se a necessidade de integração entre os setores de custo e engenharia de produtos, com o propósito de reduzir o tempo de trabalho e retrabalho.

O tema-problema dessa pesquisa é "como desenvolver uma ferramenta de parametrização de um projeto mecânico/estrutural e custo de uma chaminé de gases"?

A hipótese dessa pesquisa é a de que é possível desenvolver o respectivo projeto em um tempo bem menor, desde que os setores de custos e engenharia trabalhem em conjunto.

O tema foi escolhido considerando-se a emergente necessidade de se trabalhar com equipes multidisciplinares nas indústrias, a fim de que tanto a empresa como os funcionários sejam "um time formado por diferentes tipos de profissionais, que possuem habilidades técnicas diversas, perfis comportamentais variados, vivências e experiências distintas um dos outros". Assim, a "troca de experiências entre os profissionais será muito maior, fazendo com que eles consigam expandir seus conhecimentos" (SÓLIDES, 2020). No caso desse estudo, essa equipe é o departamento de custos e o departamento de engenharia de produtos, ou seja, composta por pessoas com diferentes habilidades, mas que possuem independência na execução e na resolução de problemas.

\section{CHAMINÉ DE GASES DE CALDEIRAS}




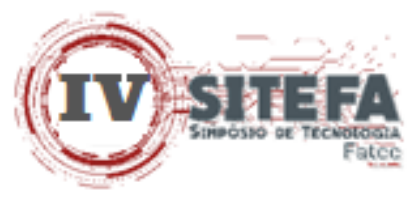

Com a crescente preocupação com os impactos ambientais, alguns métodos para minimizar esses impactos foram criados ao longo da história. Um desses meios era a construção de chaminé para retirada de gases de combustões gerados por máquinas termodinâmicas.

Essas chaminés, antigamente, eram construídas em alvenaria, e demandavam muito tempo para a construção e, além disso, demandava, também, grandes quantidades de materiais cerâmicos para a fabricação delas (BOTTI, 2004).

Com o tempo, as construções civis dessas chaminés ficaram obsoletas e foram substituídas para construções mecânicas, e reduziu-se o tempo de construção e custo de fabricação. Para reduzir o impacto ambiental é de extrema importância uma consulta ao órgão responsável da região onde a chaminé é instalada, pois ele indicará como deverá ser elaborado o estudo de dispersão de gases, e esse estudo definirá a altura mínima necessária para construção da chaminé (BOTTI, 2004).

Em regiões muito habitadas deve-se ter um cuidado especial em relação à dispersão dos gases na atmosfera e, por isso, é importante uma análise das razões dinâmicas e relativas dos gases para a elaboração do projeto (BOTTI, 2004).

As figuras 1 e 2 ilustram os tipos de chaminés.

Figura 1 - Chaminé em Aço

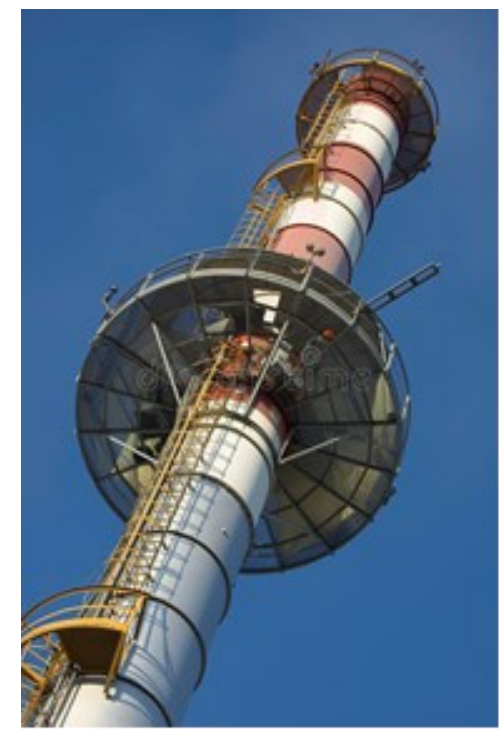

Fonte: Dreamstime (2020)

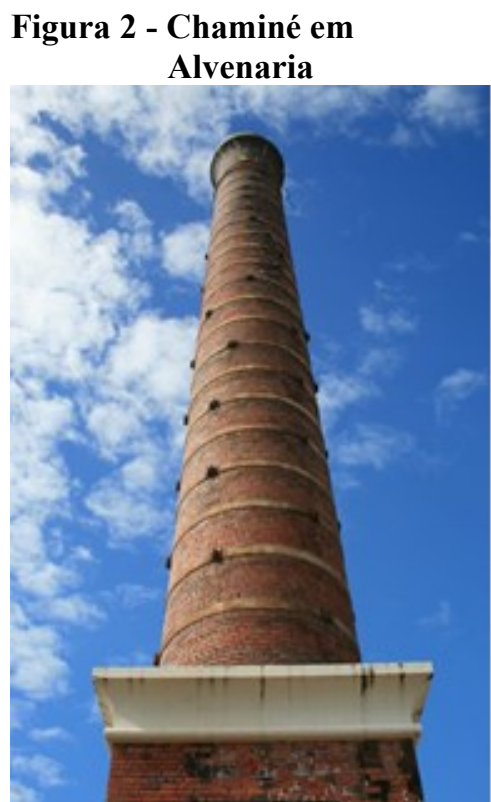

Fonte: Ross River Meatworks Chimney (2009)

\subsection{Parametrização de um projeto mecânico}

Para a parametrização de projetos mecânicos com o software Autodesk Inventor, podese utilizar planilhas eletrônicas do software Microsoft Excel com inputs iniciais, pois eles podem resolver equações e relações entre essas equações e o software Autodesk Inventor. Cria-se, assim, parâmetros que alimentarão um modelo CAD 3D no software Autodesk Inventor, com os respectivos valores inseridos na planilha do software Microsoft Excel e, com isso, o modelo reconhece essas informações e faz as devidas alterações na geométrica, no modelo (COSTA, 2016). 


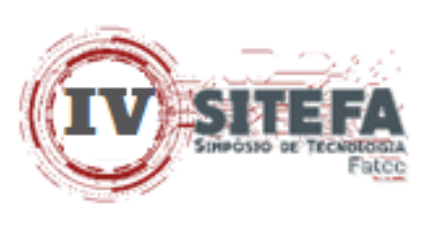

A significativa vantagem de se realizar uma parametrização geométrica está em conseguir grandes variedades de modelos de produtos com dimensões variadas em um pequeno espaço de tempo. Nesse método, o modelo se altera automaticamente devido a relação entre o modelo e a dimensão parametrizada (SPECK, 2005).

O método da parametrização dimensional reduz o tempo de modelagem e, com simples alterações básicas das dimensões, é possível gerar sólidos automaticamente (BALASTRERO JUNIOR, 2010).

Com a difusão da parametrização na indústria, ela se tornou uma ferramenta de grande valia, pois, por meio dela, se obtém a redução de custo em relação a engenheiros e técnicos. A redução de tempo pode alcançar $60 \%$ do projeto (PERES; HAYANA; VELASCO, 2007).

\subsection{Parametrização de custos}

Para definição do custo de matérias-primas e serviços são utilizadas planilhas do software Microsoft Excel, que consiste na alimentação de dados pré-definidos pelo usuário, em que eles representam descritivos de materiais e serviços aplicáveis no projeto. Além de trazer valores de custos que são atualizados com frequência, ela está totalmente parametrizada para que o usuário possa reduzir o tempo gasto para o levantamento de custos e, consequentemente, reduz o tempo de entrega do orçamento e o gasto financeiro.

O método tradicional de levantamento de materiais é elaborado pela interpretação pessoal do orçamentista, em que cada levantador tem seu próprio método, podendo, assim, um mesmo projeto ter custos diferentes e, devido a essa interpretação, dificulta a conferência do orçamento realizado (MONTEIRO; MARTINS, 2013).

A principal etapa do orçamentista é o levantamento quantitativo, no qual demanda a interpretação de projeto para a extração de informações como dimensões, massas, pesos, materiais entre outros, extraindo, assim, as informações necessárias de forma manual para compor a planilha orçamentária (WISNESKI, 2019).

\section{PROCEDIMENTOS METODOLÓGICOS}

Essa pesquisa caracteriza-se como bibliográfica e aplicada. Por meio dessa pesquisa, houve o estudo e a realização de um projeto mecânico e de custos de uma chaminé de gases, com a finalidade da interação entre os setores de engenharia e custos, buscando uma sinergia entre eles, com o propósito de otimizar o tempo e o custo.

Assim, o presente artigo teve o intento de pesquisar sobre a elaboração de um estudo de um projeto mecânico e de custos de uma chaminé de gases, sendo que a pesquisa começou a partir da idealização de um trabalho de uma equipe multidisciplinar que envolveu os setores de engenharia e custos para propor o projeto que poderá ser executado. Ressalta-se que o objeto dessa pesquisa, assim como a sua delimitação, é o estudo e desenvolvimento do projeto, não sua implementação prática.

\subsection{Microsoft Office Excel}

Na década de 80 foi lançada a primeira versão do Excel, e no ano de 1987 com a sua incorporação ao Microsoft Office tornou-se, assim, a empresa líder no desenvolvimento desse tipo de software. 


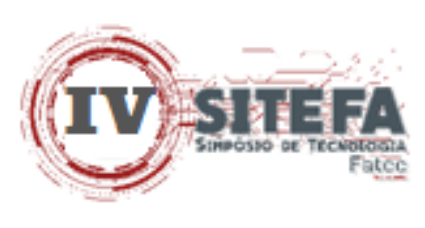

O software Microsoft Office Excel é uma ferramenta muito utilizada na atualidade, pois ela possui vários recursos em que o usuário pode elaborar tabelas, gráficos e cálculos complexos de acordo com a necessidade (LIMA, 2010).

\subsection{Autodesk Inventor}

Segundo Waguespack (2014), o software Autodesk Inventor é aplicado em projetos de engenharia em geral e, para tal, possui diversos recursos, que auxiliam na análise estrutural, modelagem e detalhamento de conjuntos e seus componentes, através de uma interface com comandos de fácil acesso para auxiliar o usuário durante o desenvolvimento do projeto.

Segundo Hercos (2015), dentre as diversas funcionalidades está a modelagem em 3D, por meio da qual é possível projetar componentes, montar conjuntos e verificar as interferências entre componentes. A integração de várias ferramentas em um único software otimiza o projeto, pois reduz os custos.

O software Autodesk Inventor permite a criação de modelos virtuais 3D que poderão, posteriormente, ser transformados em equipamentos manufaturados. Ele contempla não somente a modelagem desses modelos, mas, também, é possível avaliar os comportamentos mecânicos e estruturais (HERCOS, 2015).

\subsection{Parametrização}

Além das diversas ferramentas descritas, o software Autodesk Inventor 2020 possui ferramentas de parametrização com o objetivo de possibilitar a automatização e padronização de projetos similares, gerando uma redução no tempo e custo desses projetos. Cabe destacar que esse software cria, de forma automática, para cada dimensão inserida, um parâmetro que pode ser visualizado e modificado, diretamente ou indiretamente, o que agiliza o processo de desenvolvimento do paramétrico (HERCOS, 2015).

\section{RESULTADOS E DISCUSSÃO}

Para realizar essa pesquisa aplicada foi elaborada uma planilha com o nome Básica, na qual indica-se todas as informações necessárias para realizar o pré-projeto mecânico dentro do software Autodesk Inventor.

Todas as informações indicadas na planilha 'Básica' são correlacionadas diretamente como software Autodesk Inventor, através de parâmetros que geram a modelagem 3D do software.

Os dados que são indicados na planilha Básica alteram simultaneamente as dimensões que são necessárias para o levantamento de materiais para o orçamento da chaminé. Tais informações são indicadas dentro de uma segunda planilha chamada 'Características Dimensionais' que alimentam os dados necessários para os cálculos de pesos e áreas necessárias para o levantamento de custos.

Todas as planilhas trabalham com funções matemáticas definidas pelo usuário utilizando cálculos que definem pesos de matérias primas, especificação de materiais, tempos de fabricações, áreas de pintura, valor de matéria prima e mão de obra.

Na planilha Básica são alimentadas as seguintes informações:

- Diâmetro interno do corpo.

- Diâmetro da base. 


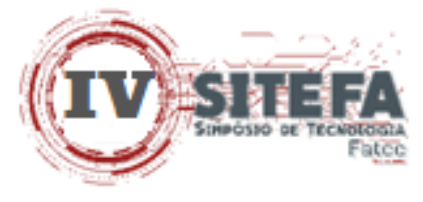

- Diâmetro cone de inferior.

- Diâmetro cone de superior.

- Altura da chaminé acabada.

- $\quad$ Altura da base.

- $\quad$ Altura do cone inferior.

- $\quad$ Altura do cone superior.

- $\quad$ Espessuras das chapas das virolas.

- $\quad$ Espessuras das chapas da base.

- $\quad$ Espessura do cone inferior.

- $\quad$ Espessura do cone superior.

- $\quad$ Quantidades de chumbadores.

- Diâmetros furos chumbadores.

\subsection{Aba 1 - Básica}

Contém informações necessárias para o pré-projeto da chaminé, todas as informações indicadas na planilha Básica (figura 3) são correlacionadas diretamente como software Autodesk Inventor através de parâmetros que geram a modelagem 3D.

\begin{tabular}{|c|c|c|c|c|c|}
\hline 4 & A & B & C & D & E \\
\hline 1 & Virolas_Diametro_Interno & 1500 & mm & & \\
\hline 2 & Virola_1_Altura & 5000 & $\mathrm{~mm}$ & & \\
\hline 3 & Virola_2_Altura & 2000 & $\mathrm{~mm}$ & & \\
\hline 4 & Virola_3_Altura & 1000 & $\mathrm{~mm}$ & & \\
\hline 5 & Virola_4_Altura & 1000 & $\mathrm{~mm}$ & & \\
\hline 6 & Virola_5_Altura & 1000 & $\mathrm{~mm}$ & & \\
\hline 7 & Virola_6_Altura & 1000 & $\mathrm{~mm}$ & & \\
\hline 8 & Virola_1_Espessura & 10 & $\mathrm{~mm}$ & & \\
\hline 9 & Virola_2_Espessura & 10 & $\mathrm{~mm}$ & & \\
\hline 10 & Virola_3_Espessura & 10 & $\mathrm{~mm}$ & & \\
\hline 11 & Virola_4_Espessura & \begin{tabular}{c|c|c|}
10 & -1 \\
\end{tabular} & $\mathrm{~mm}$ & & \\
\hline 12 & Virola_5_Espessura & 10 & $\mathrm{~mm}$ & & \\
\hline 13 & Virola_6_Espessura & 10 & $\mathrm{~mm}$ & & \\
\hline 14 & Chapa_Base_Inferior_Diametro_Externd & 2600 & $\mathrm{~mm}$ & & \\
\hline 15 & Chapa_Base_Inferior_Espessura & 25 & $\mathrm{~mm}$ & & \\
\hline 16 & Chapa_Base_Superior_Diametro_Extern & 2600 & $\mathrm{~mm}$ & & \\
\hline 17 & Chapa_Base_Superior_Espessura & 25 & $\mathrm{~mm}$ & & \\
\hline 18 & Chapa_Base_Furacao_Diametro & 2500 & $\mathrm{~mm}$ & & \\
\hline 19 & Chapa_Base_Furacao_Diametro_Furo & $50 \mid$ & $\mathrm{mm}$ & & \\
\hline 20 & Chapa_Base_Furacao_Furo_Quantidad & 20 & ul & & \\
\hline 21 & Nervura_Altura & 300 & $\mathrm{~mm}$ & & \\
\hline 22 & Nervura_Largura & 300 & $\mathrm{~mm}$ & & \\
\hline 23 & Nervura_Espessura & 10 & $\mathrm{~mm}$ & & \\
\hline 24 & Nervura_Quantidade & 20 & ul & & \\
\hline 25 & Cone_Saida_Diametro_Interno & 1000 & $\mathrm{~mm}$ & & \\
\hline 26 & Cone_Saida_Altura & 500 & $\mathrm{~mm}$ & & \\
\hline 27 & Cone_Saida_Espessura & $10 \mid$ & $\mathrm{mm}$ & & \\
\hline \multicolumn{6}{|c|}{28} \\
\hline \multicolumn{6}{|l|}{29} \\
\hline 14 & 1. Básica Características Dime & nais & Cust & & \\
\hline
\end{tabular}

Fonte: elaborada pelos autores (2020) 


\section{(IV) SITEFA}

\subsection{Aba 2 - Características Dimensionais}

Contém informações resumidas da Aba 1, essas informações estão todas correlacionadas, assim qualquer alteração na Aba 1 Básica, terá alterações simultaneamente na planilha Aba 2 'Características Dimensionais', conforme figura 4.

Figura 4 - Planilha Características Dimensionais

\begin{tabular}{|c|c|c|c|c|c|c|c|}
\hline$\Delta$ & A & & B & C & D & $\mathbf{E}$ & $\mathbf{F}$ \\
\hline 1 & & & & & & & \\
\hline 2 & & \multicolumn{3}{|c|}{ Componente } & \multicolumn{3}{|c|}{ Características Dimensionais } \\
\hline 3 & & & Descrição & Qt. & Espessura [mm] & Dimensão 1 [mm] & Dimensão 2 [mm] \\
\hline 4 & & & Virola 1 & 5 & 10 & 5000 & 4744 \\
\hline 5 & & & Virola 2 & 1 & 10 & 2000 & 4744 \\
\hline 6 & & & Virola 3 & 1 & 10 & 1000 & 4744 \\
\hline 7 & & & Virola 4 & 1 & 8 & 1000 & 4738 \\
\hline 8 & & & Virola 5 & 1 & 10 & 1000 & 4744 \\
\hline 9 & & & Virola 6 & 1 & 10 & 1000 & 4744 \\
\hline 10 & & & Chapa Base Inferior & 1 & 25 & 2600 & 1520 \\
\hline 11 & & & Chapa Base Superior & 1 & 25 & 2600 & 1520 \\
\hline 12 & & & Nervuras & 20 & 10 & 300 & 300 \\
\hline 13 & & & Cone Saida & 1 & 10 & 1500 & 4744 \\
\hline \multicolumn{8}{|l|}{14} \\
\hline \multicolumn{8}{|l|}{15} \\
\hline \multicolumn{8}{|l|}{16} \\
\hline \multicolumn{8}{|l|}{17} \\
\hline \multicolumn{8}{|l|}{18} \\
\hline \multicolumn{8}{|l|}{19} \\
\hline \multicolumn{8}{|l|}{20} \\
\hline \multicolumn{8}{|l|}{21} \\
\hline \multicolumn{8}{|l|}{22} \\
\hline \multicolumn{8}{|l|}{23} \\
\hline \multicolumn{8}{|l|}{24} \\
\hline \multicolumn{8}{|l|}{25} \\
\hline 26 & & & & & & & \\
\hline 14 & r & 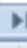 & Caracteri & cas & ensionais Custo & s $\angle$ Planilha Detalh & lada \\
\hline
\end{tabular}

Fonte: elaborada pelos autores (2020)

\subsection{Aba 3 - Custos}

Nessa Aba estão as informações nas quais foram determinados os custos de matéria prima, Aço Carbono SA-36, Aço Carbono SAC-350, Aço Inox 410-D, Aço Inox 316, mão de obra de fabricação, mão de obra para pintura.

Para determinar o custo de 'Hora Homem', foi utilizada como base de produtividade a especificação da matéria prima para Aço Carbono SAC-350, na pintura foi considerado o custo e rendimento teórico por $\mathrm{m}^{2}$ para a mesma matéria prima.

Na Aba 3 contém um filtro em cada coluna "SIM / NÃO", sendo possível de forma rápida determinar itens a serem considerados no projeto final. 


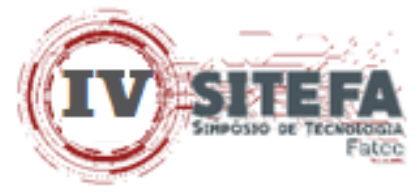

As informações pré-definidas na Aba 2 estão vinculadas com a Aba $\mathbf{3}$ tendo, assim, o levantamento da massa total do equipamento, através de cálculos, e entre tais informações obteve-se um custo final estimado da chaminé, indicada na figura 5.

Ressalta-se que todos os custos pré-definidos na planilha Aba 3 podem ser alterados com base em valores atualizados de mercado, tais custos foram considerados já processados para cada item, conforme mencionados anteriormente.

Figura 5 - Planilha Custos

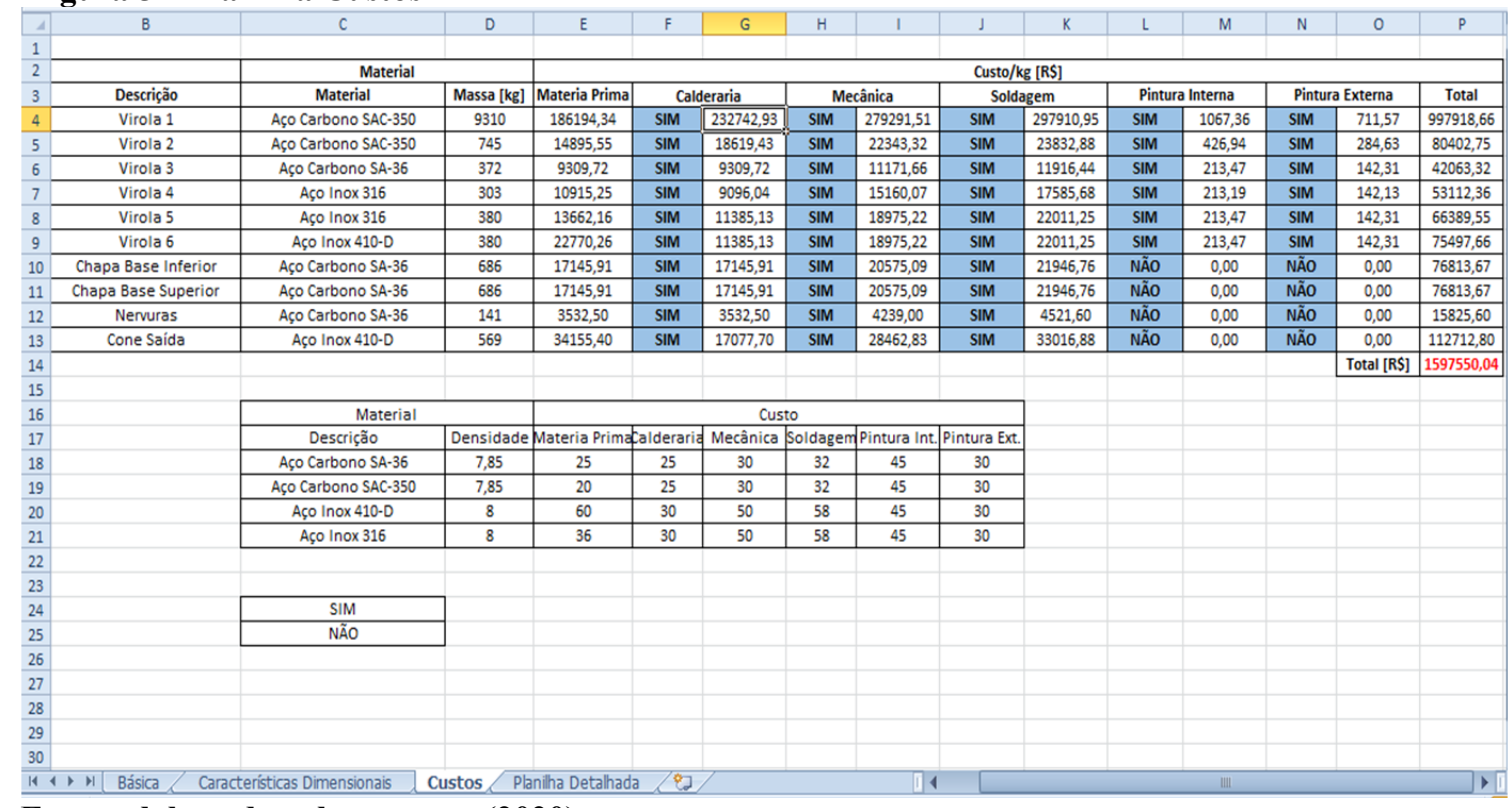

Fonte: elaborada pelos autores (2020)

\subsection{Aba 4 - Planilha Detalhada}

Através dessa Aba obtém-se um resumo em relação à quantidade de massa a ser considerada para a fabricação da Chaminé, considerando-se especificação e espessura da matéria prima a ser utilizada, representada na figura 6 .

Figura 6 - Planilha Detalhada

\begin{tabular}{|c|c|c|c|c|c|c|c|}
\hline 2 & A & B & C & D & $\mathrm{E}$ & $\mathrm{F}$ & G \\
\hline \multicolumn{8}{|l|}{1} \\
\hline 2 & QT. & DESCRIÇÃO DO MATERIAL & Esp. (mm) & Massa [kg] & & DESCRIÇÃO DO MATERIAL & Massa [kg] \\
\hline 3 & & COSTADO & & & & Aço Carbono SA-36 & 1885 \\
\hline 4 & 10 & Aço Carbono SAC-350 & 5 & 9279 & & Aço Carbono SAC-350 & 10024 \\
\hline 5 & 1 & Aço Carbono SAC-350 & 10 & 745 & & Aço Inox 410-D & 776 \\
\hline 6 & 1 & Aço Carbono SA-36 & 10 & 372 & & Aço Inox 316 & 683 \\
\hline 7 & 1 & Aço Inox 316 & 8 & 303 & & & \\
\hline 8 & 1 & Aço Inox 316 & 10 & 380 & & MASSA TOTAL & 13368 \\
\hline 9 & 1 & Aço Inox 410-D & 10 & 380 & & & \\
\hline 10 & & BASE DE SUSTENTAÇÃO & & & & & \\
\hline 11 & 1 & Aço Carbono SA-36 & 25 & 686 & & & \\
\hline 12 & 1 & Aço Carbono SA-36 & 25 & 686 & & & \\
\hline 13 & 20 & Aço Carbono SA-36 & 10 & 141 & & & \\
\hline 14 & 1 & Aço Inox 410-D & 10 & 397 & & & \\
\hline
\end{tabular}

Fonte: elaborada pelos autores (2020) 


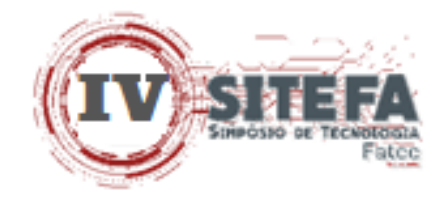

\subsection{Ferramenta Parâmetros no Software Autodesk Inventor}

A forma mais simples de parametrizar é através da ferramenta parameters, indicada na figura 7, que pode ser acessada através do menu manager, onde estão os parâmetros relativos a todas as dimensões utilizadas na construção do componente ou do conjunto, criadas automaticamente pelo software.

Figura 7 - Ferramenta Parameters

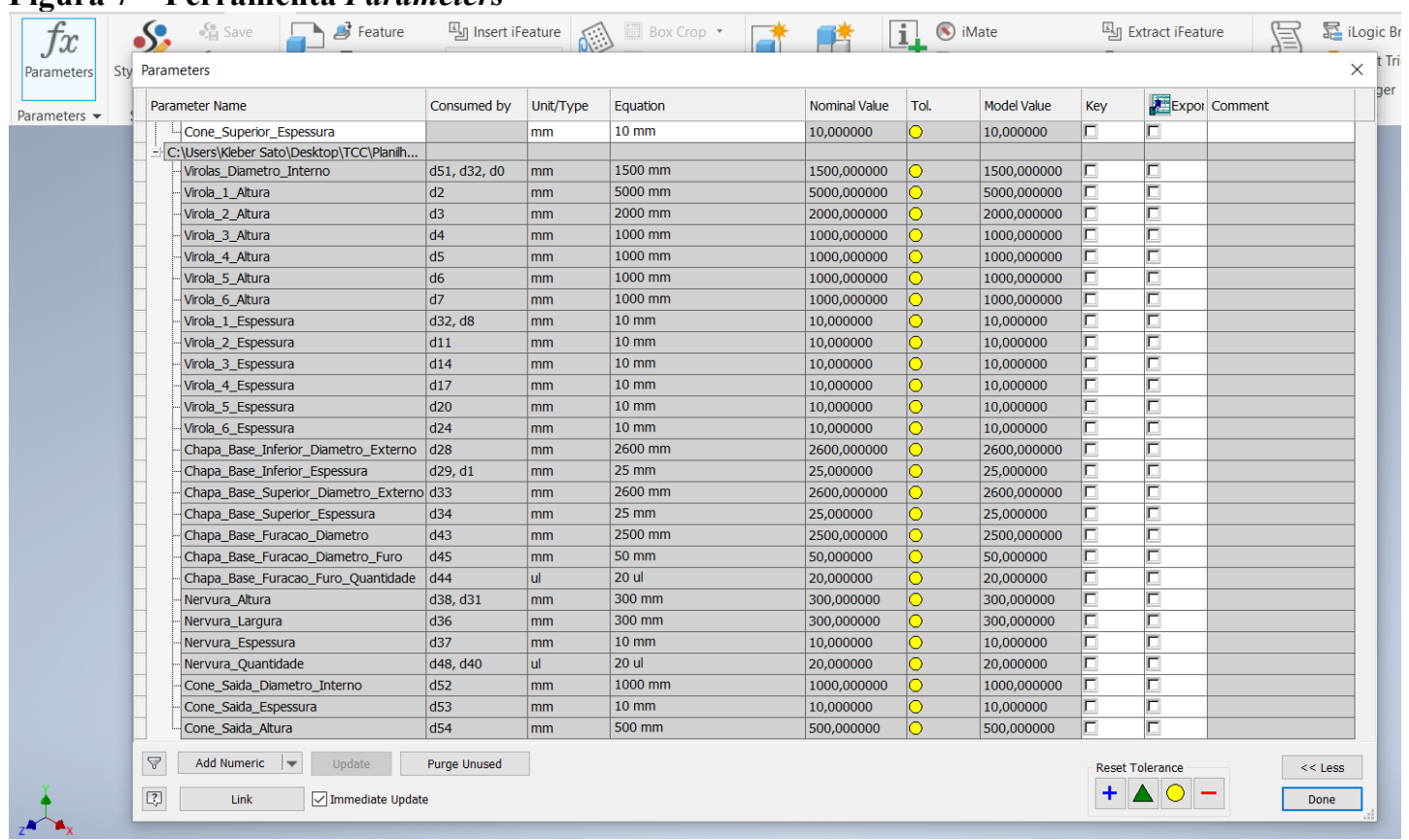

Fonte: elaborada pelos autores (2020)

No recurso utilizado dessa ferramenta para elaboração de simulação de modelos foi utilizada a ferramenta chamada parameters, na qual importa os dados do Excel para dentro do software. Dentro do Autodesk Inventor também foram utilizadas ferramentas de modelagem como extrude indicada na figura 8.

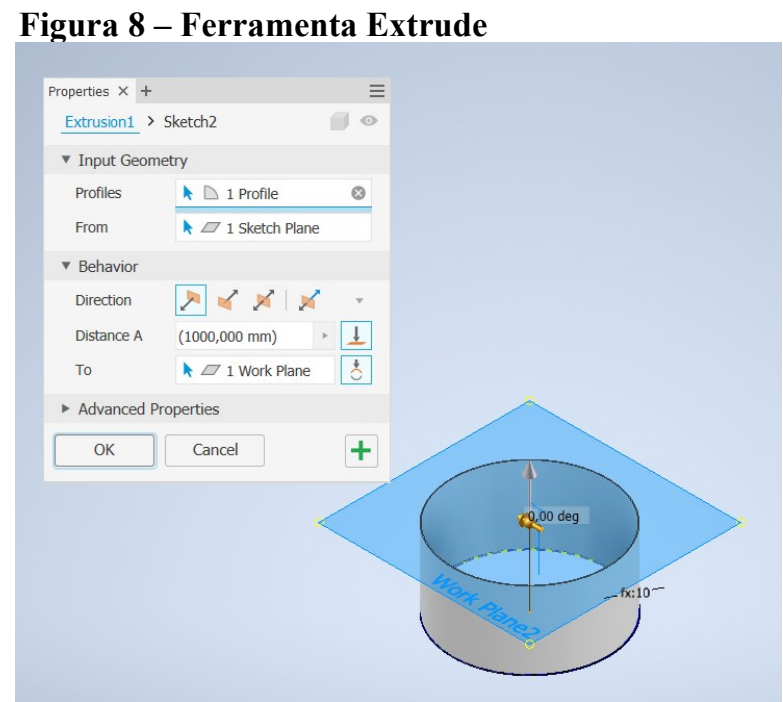

Fonte: elaborada pelos autores (2020) 


\section{STIEFA}

Fatce

A utilização da ferramenta revolve do Autodesk Inventor, resulta na modelagem conforme figura 9.

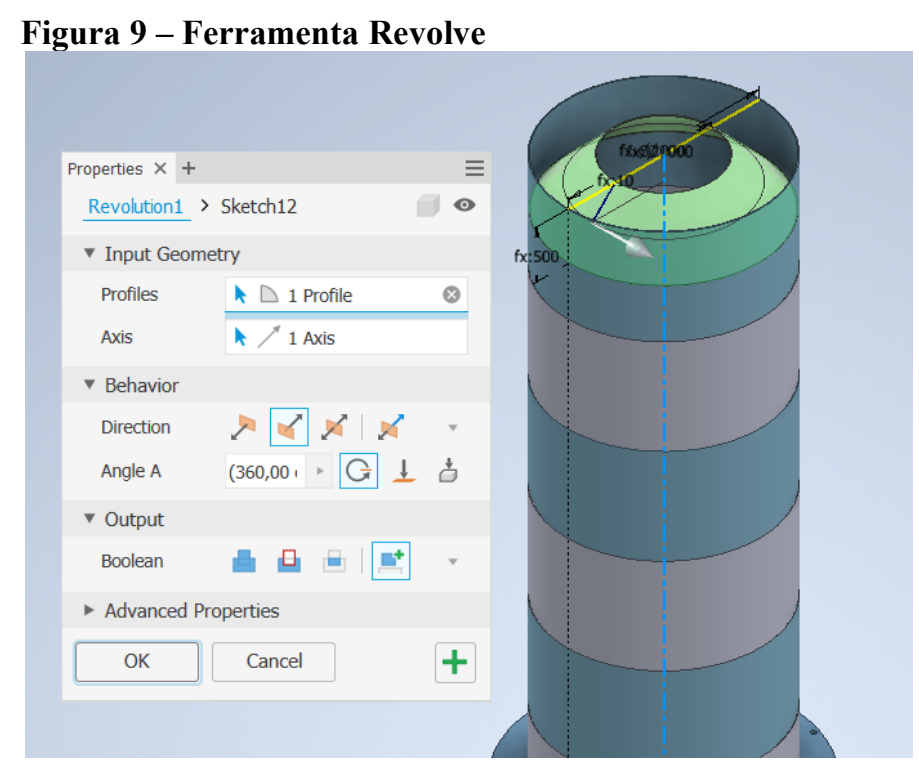

Fonte: elaborada pelos autores (2020)

Com a utilização da ferramenta maneger é possível alterar valores e inserir relações e equações cujos resultados são reproduzidos automaticamente no modelo $3 \mathrm{D}$, como é apresentado na figura 10 .

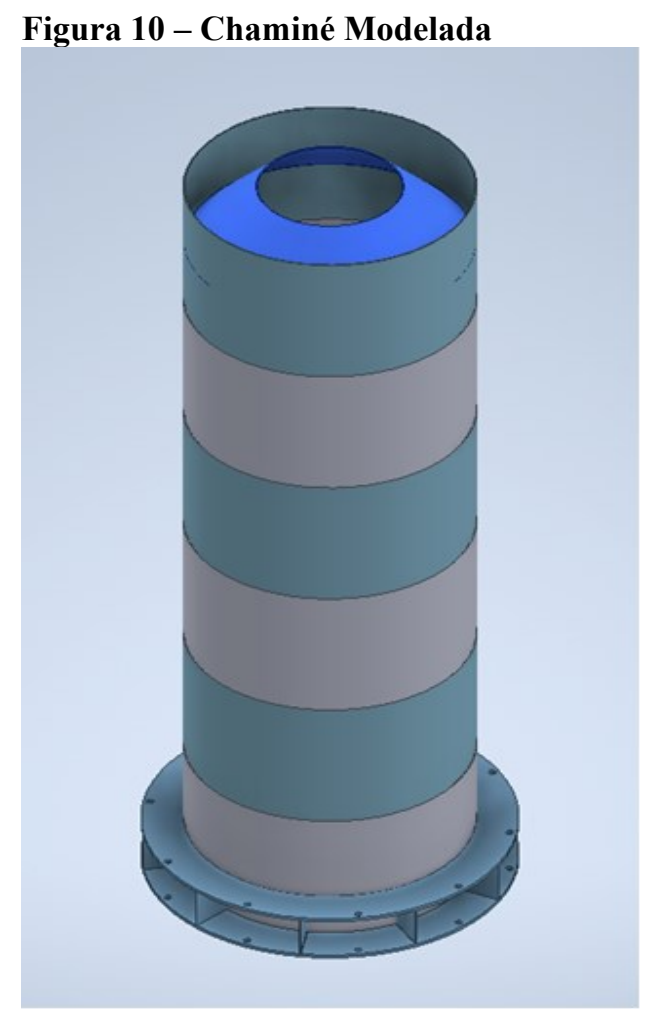

Fonte: elaborada pelos autores (2020) 


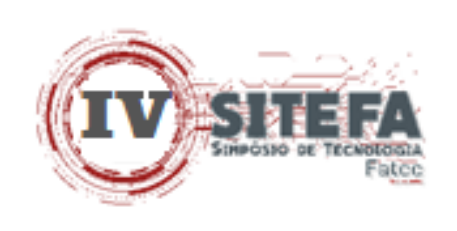

\section{CONCLUSÃO}

Esse artigo apresentou os resultados de uma pesquisa que visou o desenvolvimento de um projeto de parametrização e custo de uma chaminé de gases. Os resultados da análise dos dados ratificam o que é apresentado na teoria: a grande importância das informações de custos no processo de parametrização, tanto para o ganho de produtividade quanto para o ganho de tempo no projeto.

No programa desenvolvido com esse trabalho foram utilizados vários parâmetros para o dimensionamento da chaminé e, com isso, o programa se mostrou eficiente para levantamento de custo estimado e modelagem.

Para o método de levantamento tradicional leva em torno de 4 a 5 dias para o estudo do projeto, levantamento de materiais e levantamento de custos.

Contudo, aplicando-se o método após o desenvolvimento dessa ferramenta, esse tempo é reduzido para em torno de 1 a 2 horas, o que é bastante significativo.

Para futuras pesquisas sugere-se a aplicação em equipamentos com formas construtivas diferentes como, por exemplo, um tanque de armazenamento. Sugere-se, ainda, fazer a modelagem para confrontar os resultados teóricos com os resultados práticos.

\section{REFERÊNCIAS}

BALASTRERO JUNIOR, José Orlando. Modelagem e otimização de componentes mecânicos em sistemas CAD 3D com o emprego de geometrias paramétricas, 2010. Disponível em: http://repositorio.unitau.br/jspui/bitstream/20.500.11874/673/1/Jose\%20Orlando\%20Balastrer o\%20Jr.pdf Acesso em: 18 dez. 2020.

BOTTI, H. N., Estudo sobre o desenvolvimento de projeto de chaminé. Centro Tecnológico da Universidade Federal do Espírito Santo, 2004. Disponível em: https://engenhariamecanica.ufes.br/sites/engenhariamecanica.ufes.br/files/field/anexo/20041_-_henrique_negri_botti.pdf Acesso em: 18 dez. 2020.

COSTA, M. A., Parametrização e cálculo estrutural através do método dos elementos finitos de uma comporta segmento de fundo. Guaratinguetá, 2016. Disponível em: https://repositorio.unesp.br/bitstream/handle/11449/144230/costa_ma_me_guara.pdf?sequenc $\mathrm{e}=3$ \&isAllowed=y Acesso em: 18 dez. 2020.

DREAMSTIME. Chaminé em Aço 2020. Disponível em: https:/c8.alamy.com/comp/DM27TY/detail-of-an-high-chimney-in-a-modern-industrialplant-DM27TY.jpg. Acesso em: 18 dez. 2020.

HERCOS, L. R. P. Projeto estrutural e parametrização de uma comporta deslizante utilizando o software Autodesk Inventor. 2015. 115f. Dissertação de Mestrado em Engenharia Mecânica - Faculdade de Engenharia do Campus de Guaratinguetá, Universidade Estadual Paulista, Guaratinguetá, 2015. Disponível em: https://repositorio.unesp.br/bitstream/handle/11449/124393/000832833.pdf?sequence=1\&isAl lowed=y Acesso em: 18 dez. 2020. 
LIMA, R. et al. A história do Microsoft Office Excel. 2010. Disponível em:

http://planilhaeletro.blogspot.com.br/2010/10/historia-do-microsoft-officeexcel.html Acesso em: 15 jan. 2020.

MONTEIRO, André; MARTINS, João Poças. A survey on modeling guidelines for quantity takeoff-oriented BIM-based design. Automation in Construction, v. 35, p. 238253, 2013.

Disponível em: https://repositorio-aberto.up.pt/handle/10216/96525 Acesso em: 18 dez. 2020.

PERES, M. P.; HAYANA, A. O. F.; VELASCO, A. D.. A Parametrização e a Engenharia: Curitiba, 2007. Disponível em:

http://www.exatas.ufpr.br/portal/docs_degraf/artigos_graphica/APARAMETRIZACAOEAE NGENHARIA.pdf Acesso em: 18 dez. 2020.

ROSS RIVER MEATWORKS CHIMNEY. Chaminé em alvenaria. 2009. Disponível em: https://en.wikipedia.org/wiki/Ross_River_Meatworks_Chimney. Acesso em: 18 dez. 2020.

SPECK, H. J.. Proposta de método para facilitar a mudança das técnicas de projetos: da prancheta à modelagem sólida $(\mathrm{CAD})$ para empresas de engenharia de pequeno e médio porte. Florianópolis, 2005. Disponível em:

https://repositorio.ufsc.br/bitstream/handle/123456789/102197/221294.pdf?sequence=1\&isAl lowed=y Acesso em: 18 dez. 2020.

SÓLIDES. O que é uma equipe multidisciplinar e quais são os seus benefícios. 2020. Disponível em: https://blog.solides.com.br/equipe-multidisciplinar/. Acesso em: 11 maio 2021.

WAGUESPACK, C. Mastering Autodesk Inventor 2014, and Autodesk Inventor LT. 2014.

WISNESKI, C.. Análise comparativa do levantamento de quantitativos de serviço entre o método tradicional e a tecnologia BIM. 2019. Disponível em:

https://bibliodigital.unijui.edu.br:8443/xmlui/bitstream/handle/123456789/6436/Catia\%20Pes \%20Wisneski.pdf?sequence=1\&isAllowed=y Acesso em: 18 dez. 2020. 